IMAFF-2/93

February 1993

\title{
The Hidden Quantum Group of the 8-vertex Free Fermion Model: q-Clifford Algebras
}

\author{
R. Cuerno, C. Gómez, E. López-Manzanares and G. Sierra \\ Instituto de Matemáticas y Física Fundamental, CSIC \\ Serrano 123, E-28006 Madrid, SPAIN
}

\begin{abstract}
We prove in this paper that the elliptic $R$-matrix of the eight vertex free fermion model is the intertwiner $R$-matrix of a quantum deformed Clifford-Hopf algebra. This algebra is constructed by affinization of a quantum Hopf deformation of the Clifford algebra.
\end{abstract}




\section{Introduction}

The realm of two dimensional integrable models contains two important families associated to the six vertex and eight vertex solutions to the Yang-Baxter equation [1].

Whereas the family of six vertex solutions (six vertex model and their higher spin descendants) are $R$-matrix intertwiners for different finite dimensional irreducible representations of $U_{q}(\widehat{S l(2)})$, the elliptic eight vertex solutions do not admit, for the time being, the interpretation as quantum group intertwiners. To find such a quantum group interpretation of the eight vertex model would provide, for instance, a natural way to extend the known hidden quantum group structure of conformal field theories [2] to $q$-conformal field theories defined by the $q$-deformed Knizhnik-Zamolodchikov equation [3].

A special class of solutions to the vertex Yang-Baxter (YB) equation are the ones satisfying the so called free fermion condition:

$$
R_{00}^{00}(u) R_{11}^{11}(u)+R_{01}^{10}(u) R_{10}^{01}(u)=R_{00}^{11}(u) R_{11}^{00}(u)+R_{01}^{01}(u) R_{10}^{10}(u)
$$

In the six vertex case, $R_{00}^{11}(u)=R_{11}^{00}(u)=0$ the solutions to YB satisfy (1) and are given by the $R$-matrix intertwiners of the Hopf subalgebra $U_{\hat{q}}(g l \widehat{(1,1)})$ [ [4]. These intertwiners can be mapped into the ones of $U_{q}(\widehat{s l(2)})\left(q^{4}=1\right)$ for non classical nilpotent irreducible representations [5] with $\hat{q}=\lambda$ and $\lambda^{2}$ the eigenvalue of the casimir $K^{2}$. The physical interest of the free fermion six vertex solutions is their close connection with $N=2$ integrable models. In fact we can define using the generators of $U_{q}(\widehat{s l(2)})$ for $q^{4}=1$ a $N=2$ supersymmetric algebra [6], and in this case the free fermion condition (11) reflects the $N=2$ invariance of the $R$-matrix. Moreover the $N=2$ piece of the solitonic $S$-matrix for the $N=2$ Ginzburg-Landau superpotential $W=X^{N+1} /(N+1)-\beta X$ [7] can be shown to be given by the intertwiners of $U_{\hat{q}}(g l \widehat{(1,1)})$ with $\hat{q}^{N}=1$.

In the eight vertex case, $R_{00}^{11}(u) \neq 0 R_{11}^{00}(u) \neq 0$, solutions to YB satisfying (11) have been known for a long time [8]. The most general solution corresponding to imposing non-zero field [9], [10] depends on three spectral parameters $u, \psi_{1}, \psi_{2}$, and is given by:

$$
\begin{aligned}
a & \equiv R_{00}^{00}=1-e(u) e\left(\psi_{1}\right) e\left(\psi_{2}\right) \\
\tilde{a} & \equiv R_{11}^{11}=e(u)-e\left(\psi_{1}\right) e\left(\psi_{2}\right) \\
b & \equiv R_{01}^{10}(u)=e\left(\psi_{1}\right)-e(u) e\left(\psi_{2}\right) \\
\tilde{b} & \equiv R_{10}^{01}=e\left(\psi_{2}\right)-e(u) e\left(\psi_{1}\right) \\
c & \equiv R_{01}^{01}=R_{10}^{10}=\left(e\left(\psi_{1}\right) \operatorname{sn}\left(\psi_{1}\right)\right)^{1 / 2}\left(e\left(\psi_{2}\right) \operatorname{sn}\left(\psi_{2}\right)\right)^{1 / 2}(1-e(u)) / \operatorname{sn}(u / 2) \\
d & \equiv R_{00}^{11}=R_{11}^{00}=-i k\left(e\left(\psi_{1}\right) \operatorname{sn}\left(\psi_{1}\right)\right)^{1 / 2}\left(e\left(\psi_{2}\right) \operatorname{sn}\left(\psi_{2}\right)\right)^{1 / 2}(1+e(u)) \operatorname{sn}(u / 2)
\end{aligned}
$$


with $e(u)$ the elliptic exponential:

$$
e(u)=c n(u)+i \sin (u)
$$

and $k$ the elliptic modulus. The Yang-Baxter equation satisfied by this $R$ matrix is [10]:

$$
\begin{aligned}
& \left(\mathbf{1} \otimes R\left(u ; \psi_{1}, \psi_{2}\right)\right)\left(R\left(u+v ; \psi_{1}, \psi_{3}\right) \otimes \mathbf{1}\right)\left(\mathbf{1} \otimes R\left(v ; \psi_{2}, \psi_{3}\right)\right)= \\
& \left(R\left(v ; \psi_{2}, \psi_{3}\right) \otimes \mathbf{1}\right)\left(\mathbf{1} \otimes R\left(u+v ; \psi_{1}, \psi_{3}\right)\right)\left(R\left(u ; \psi_{1}, \psi_{2}\right) \otimes \mathbf{1}\right)
\end{aligned}
$$

The simplest way to catch the physical meaning of solution (2) is to define the corresponding spin chain hamiltonian:

$$
H=\left.\sum_{j=1}^{N} i \frac{\partial}{\partial u} R_{j, j+1}(u ; \psi, \psi)\right|_{u=0}
$$

which is the well known $X Y$ - model in an external magnetic field [11]:

$$
H=\sum_{j=1}^{N}\left[(1+\Gamma) \sigma_{j}^{x} \sigma_{j+1}^{x}+(1-\Gamma) \sigma_{j}^{y} \sigma_{j+1}^{y}+h\left(\sigma_{j}^{z}+\sigma_{j+1}^{z}\right)\right]
$$

where:

$$
\begin{aligned}
\Gamma & =\frac{2 c d}{a b+\tilde{a} \tilde{b}}=k \operatorname{sn}(\psi) \\
h & =\frac{a^{2}+b^{2}-\tilde{a}^{2}-\tilde{b}^{2}}{2(a b+\tilde{a} \tilde{b})}=c n(\psi)
\end{aligned}
$$

In this letter and as a preliminary step of the long term process of finding the quantum group symmetry of the eight vertex model, we will define a fully fledged Hopf algebra such that its $R$-intertwiners coincide with the elliptic free fermionic eight vertex solution (2).

\section{The quantum Clifford algebra}

A Clifford algebra $C(\eta)$ associated to a cuadratic form $\eta$ is the associative algebra generated by the elements $\left\{\Gamma_{\mu}\right\}_{\mu=0}^{D}$, which satisfy:

$$
\left\{\Gamma_{\mu}, \Gamma_{\nu}\right\}=2 \eta_{\mu \nu} \mathbf{1} \quad \mu=1, \ldots, D
$$


Associated to $C(\eta)$ we define the Clifford-Hopf algebra $\mathrm{CH}(\mathrm{D})$ as the associative algebra generated by $\Gamma_{\mu} \quad(\mu=1, \ldots, D), \quad \Gamma_{D+1}$ and the central elements $E_{\mu} \quad(\mu=$ $1, \ldots, D)$ satisfying the following relations:

$$
\begin{aligned}
& \Gamma_{\mu}^{2}=E_{\mu}, \Gamma_{D+1}^{2}=\mathbf{1} \\
& \left\{\Gamma_{\mu}, \Gamma_{\nu}\right\}=0, \quad \mu \neq \nu \\
& \left\{\Gamma_{\mu}, \Gamma_{D+1}\right\}=0 \\
& {\left[E_{\mu}, \Gamma_{\nu}\right]=\left[E_{\mu}, \Gamma_{D+1}\right]=\left[E_{\mu}, E_{\nu}\right]=0 \quad \forall \mu, \nu}
\end{aligned}
$$

The algebra $\mathrm{CH}(\mathrm{D})$ is a Hopf algebra with the following comultiplication $\Delta$, antipode $S$ and counit $\epsilon$ :

$$
\begin{array}{lll}
\Delta\left(E_{\mu}\right)=E_{\mu} \otimes \mathbf{1}+\mathbf{1} \otimes E_{\mu}, & S\left(E_{\mu}\right)=-E_{\mu}, & \epsilon\left(E_{\mu}\right)=0 \\
\Delta\left(\Gamma_{\mu}\right)=\Gamma_{\mu} \otimes \mathbf{1}+\Gamma_{D+1} \otimes \Gamma_{\mu}, & S\left(\Gamma_{\mu}\right)=\Gamma_{\mu} \Gamma_{D+1}, & \epsilon\left(\Gamma_{\mu}\right)=0 \\
\Delta\left(\Gamma_{D+1}\right)=\Gamma_{D+1} \otimes \Gamma_{D+1}, & S\left(\Gamma_{D+1}\right)=\Gamma_{D+1}, & \epsilon\left(\Gamma_{D+1}\right)=1
\end{array}
$$

For $\mathrm{D}$ even the elements $E_{\mu} \quad(\mu=1, \ldots, D)$ and the product $\Gamma_{1} \cdots \Gamma_{D} \Gamma_{D+1}$ are casimirs of $\mathrm{CH}(\mathrm{D})$, therefore in an irreducible representation of $\mathrm{CH}(\mathrm{D})$ we get $E_{\mu}=\eta_{\mu \mu}$, and $\Gamma_{D+1} \sim \Gamma_{1} \cdots \Gamma_{D}$ which means that the irreps of $\mathrm{CH}(\mathrm{D})$ are isomorphic to those of $C(\eta)$ for all possible signatures of $\eta$ (there is a unique faithful representation of $C(\eta)$ of dimension $2^{D}$ ). For $\mathrm{D}$ odd similar arguments show that the representation theory of $\mathrm{CH}(\mathrm{D})$ is related to that of $C(\eta)$ for $\eta$ a quadratic form defined in one dimension higher, namely $\mathrm{D}+1$.

The quantum deformation of $\mathrm{CH}(\mathrm{D})$, that we will denote $\mathrm{CH}_{q}(\mathrm{D})$, is defined by:

$$
\Gamma_{\mu}^{2}=\left[E_{\mu}\right]_{q}=\frac{q^{E_{\mu}}-q^{-E_{\mu}}}{q-q^{-1}}
$$

with the rest of equations (9) unchanged. The comultiplication for $\Gamma_{\mu}$ is now given by:

$$
\Delta \Gamma_{\mu}=\Gamma_{\mu} \otimes q^{-E_{\mu} / 2}+q^{E_{\mu} / 2} \Gamma_{D+1} \otimes \Gamma_{\mu}
$$

The Hopf algebra $\mathrm{CH}_{q}(\mathrm{D})$ for $D=2$ is very close to the two parameter quantum supergroup $\mathcal{U}_{\alpha, \beta}(s u(1,1))$ defined in [12]. The correspondence between both algebras is given by the substitutions $q^{E_{x}} \longrightarrow \alpha^{E}$ and $q^{E_{y}} \longrightarrow \beta^{E}$. Notice that in $C H_{q}(2)$ we have two central elements $E_{x}, E_{y}$ and one quantum deformation parameter, while in $\mathcal{U}_{\alpha, \beta}(s u(1,1))$ there exist one central element and two parameters. This difference will be important in 
the representation theory. We observe that the "SUSY grading" is played in our case by $\Gamma_{3}$, and in general by $\Gamma_{D+1}$ for $D>2$.

Next we proceed to define a sort of affinization of the Hopf algebra $\mathrm{CH}_{q}(\mathrm{D})$. The generators of this new algebra that we denote $C \widehat{H_{q}(D)}$ are: $E_{\mu}^{(i)}, \Gamma_{\mu}^{(i)}, \Gamma_{D+1}^{(i)}, \quad i=0,1$ satisfying (11) and (12) for each value of $i$. In what follows we will consider only the case $D=2$.

A two dimensional irrep $\pi_{\xi}$ of $C \widehat{H_{q}(D)}$ is labelled by three complex parameters $\xi=$ $\left(z, \lambda_{x}, \lambda_{y}\right) \in C_{\times}^{3}$ and reads:

$$
\begin{gathered}
\pi_{\xi}\left(\Gamma_{x}^{(0)}\right)=\left(\frac{\lambda_{x}^{-1}-\lambda_{x}}{q-q^{-1}}\right)^{1 / 2}\left(\begin{array}{cc}
0 & z^{-1} \\
z & 0
\end{array}\right), \pi_{\xi}\left(\Gamma_{x}^{(1)}\right)=\left(\frac{\lambda_{x}-\lambda_{x}^{-1}}{q-q^{-1}}\right)^{1 / 2}\left(\begin{array}{cc}
0 & z \\
z^{-1} & 0
\end{array}\right) \\
\pi_{\xi}\left(\Gamma_{y}^{(0)}\right)=\left(\frac{\lambda_{y}^{-1}-\lambda_{y}}{q-q^{-1}}\right)^{1 / 2}\left(\begin{array}{cc}
0 & -i z^{-1} \\
i z & 0
\end{array}\right), \pi_{\xi}\left(\Gamma_{y}^{(1)}\right)=\left(\frac{\lambda_{y}-\lambda_{y}^{-1}}{q-q^{-1}}\right)^{1 / 2}\left(\begin{array}{cc}
0 & -i z \\
i z^{-1} & 0
\end{array}\right) \\
\pi_{\xi}\left(\Gamma_{3}^{(0)}\right)=\left(\begin{array}{cc}
1 & 0 \\
0 & -1
\end{array}\right), \pi_{\xi}\left(\Gamma_{3}^{(1)}\right)=\left(\begin{array}{cc}
1 & 0 \\
0 & -1
\end{array}\right) \\
\pi_{\xi}\left(q^{E_{x}^{(0)}}\right)=\lambda_{x}^{-1}, \pi_{\xi}\left(q^{E_{x}^{(1)}}\right)=\lambda_{x} \\
\pi_{\xi}\left(q^{E_{y}^{(0)}}\right)=\lambda_{y}^{-1}, \pi_{\xi}\left(q^{E_{y}^{(1)}}\right)=\lambda_{y}
\end{gathered}
$$

The intertwiner $R_{\xi_{1}, \xi_{2}}$ for two of these irreps is defined by the condition

$$
R_{\xi_{1} \xi_{2}} \Delta_{\xi_{1} \xi_{2}}(a)=\Delta_{\xi_{2} \xi_{1}}(a) R_{\xi_{1} \xi_{2}} \quad \forall a \in C \widehat{H_{q}}(2)
$$

with $\Delta_{\xi_{1} \xi_{2}}=\pi_{\xi_{1}} \otimes \pi_{\xi_{2}}(\Delta)$. Assuming that $R_{\xi_{1}, \xi_{2}}$ is an invertible matrix, then the intertwiner equation (14) implies:

$$
\operatorname{tr} \Delta_{\xi_{1} \xi_{2}}(a)=\operatorname{tr} \Delta_{\xi_{2} \xi_{1}}(a) \quad \forall a \in C \widehat{H_{q}}(2)
$$

For $a=\Gamma_{x}^{(0)} \Gamma_{y}^{(1)}$ we obtain the following constraint on the labels of the irreps which admit an intertwiner:

$$
\frac{2(\lambda-\mu)}{\left(1-\lambda^{2}\right)^{1 / 2}\left(1-\mu^{2}\right)^{1 / 2}\left(z^{2}-z^{-2}\right)}=k
$$

\footnotetext{
${ }^{1}$ To define the algebra $C \widehat{H_{q}(D)}$ properly we should add to 11 the equivalent to Serre's relations. These will not be relevant for the discussion in this paper.
} 
with $k$ an arbitrary constant. Equation (16) defines a two dimensional variety embedded in $\mathbf{C}^{3}$ which can be uniformized in terms of elliptic functions. Identifying $k$ in (16) with the elliptic modulus we define a new variable $\varphi$ by:

$$
z^{2}=\operatorname{cn}(\varphi)+i s n(\varphi)
$$

Definig now:

$$
\lambda_{x}=\tanh x, \quad \lambda_{y}=\tanh y
$$

equation (16) becomes

$$
e^{x-y}=d n(\varphi)+i k s n(\varphi)
$$

which means that $x+y$ is independent of $\varphi$, therefore each point in the curve (16) can be parametrized by two complex parameters $(\varphi, \psi)$ with $\psi$ defined by

$$
\tanh \left(\frac{x+y}{2}\right)=\operatorname{cn}(\psi)+i \sin (\psi)
$$

The main result of this paper is that, given two irreps lying on the same curve (16), $\xi_{1}\left(\varphi_{1}, \psi_{1}\right)$ and $\xi_{2}\left(\varphi_{2}, \psi_{2}\right)$ the intertwiner $R$-matrix $R_{\xi_{1}, \xi_{2}}$ coincides with the one given in (2) (up to a diagonal change of basis) provided we identify $u=\varphi_{1}-\varphi_{2}$. Notice from (17) that the "affine" parameter $z$ becomes the standard exponential in the trigonometric limit. The derivation of (2) is long but straighforward, and we have used the following identity among elliptic functions:

$$
e\left(\varphi_{1}-\varphi_{2}\right)=\frac{e\left(\varphi_{1}\right)\left(d n\left(\varphi_{1}\right)+1\right)\left(d n\left(\varphi_{2}\right)+1\right)-k^{2} e\left(\varphi_{2}\right) \operatorname{sn}\left(\varphi_{1}\right) \operatorname{sn}\left(\varphi_{2}\right)}{e\left(\varphi_{2}\right)\left(d n\left(\varphi_{1}\right)+1\right)\left(d n\left(\varphi_{2}\right)+1\right)-k^{2} e\left(\varphi_{1}\right) \operatorname{sn}\left(\varphi_{1}\right) \operatorname{sn}\left(\varphi_{2}\right)}
$$

Summarizing our results we have proved that the intertwiner $R$-matrix for two dimensional irreps of the Hopf algebra $C \widehat{H_{q}}(2)$ is the free fermion eight vertex solution to the Yang-Baxter equation.

\section{Comments}

The Sklyanin algebra [13] of the eight vertex model is determined by the corresponding elliptic curve and the anisotropy $\gamma$ [14]. In the free fermionic case, i.e. $\gamma=K$, the curve, for the most general case with non-zero field, is given by (16). An important question that we will address in a future publication, is the mathematical meaning, inside $C \widehat{H_{q}}(2)$, of the $\gamma=K$-Sklyanin algebra. 
Taking into account that the trigonometric limit of the free fermion model is given by the six vertex free fermion model and that this $R$-matrix is the intertwiner of $U_{\hat{q}}(g l(\widehat{1}, 1))$, it is plausible to conjecture that the Hopf algebra $C \widehat{H_{q}}(2)$ plays the role, in the sense of reference [3], of hidden quantum group of the $q-$ WZW model defined by $U_{q}(g l \widehat{(1,1)})$. More precisely we expect that the connection matrices of the $q-\mathrm{KZ}$ equation for $U_{q}(g l \widehat{(1,1)})$ are quantum $6-j$ symbols of $C \widehat{H_{q}}(2)$.

Another interesting issue is the interpretation of the eight vertex free fermion $R$-matrix as an scattering $S$-matrix in the sense of Zamolodchikov [15]. From our previous results we know that the correspondent "solitons" define now irreducible representations of $C \widehat{H_{q}}(2)$. Even though we cannot expect a field theory limit preserving the elliptic nature of this $S$-matrix, as a consequence of the c-theorem [16], the elliptic $S$-matrix (2) may still have a good physical meaning in the lattice, maybe related to the dynamics of the cnoidal waves in a Toda lattice [17].

Finally and based on the previously mentioned close connection between $N=2$ soliton $S$-matrices and intertwiners of $U_{q}(g l \widehat{(1,1)})$, it is natural to wonder if some relevant information on $N=2$ integrable models is still hidden in the quantum Clifford algebra $\widehat{C H_{q}}(2)$.

\section{Acknowledgements}

We would like A. Berkovich for valuable discussions. The work of R.C. and E.L.M. is supported by M.E.C. fellowships. 


\section{References}

[1] R.J.Baxter: Exactly solved models in statistical mechanics. Academic Press, 1982

[2] L.Alvarez-Gaumé, C.Gm̈ez and G.Sierra, Nucl. Phys. B319 (1989) 155, Phys. Lett. B220 (1989) 142, Nucl. Phys. B330 (1990) 347

G.Moore and N.Yu.Reshetikhin, Nucl. Phys. B328 (1989) 557

C.Gómez and G.Sierra, Nucl. Phys. B352 (1991) 791

[3] I.B.Frenkel and N.Yu.Reshetikhin, Commun. Math. Phys. 146 (1992) 1

[4] L.Rozansky and H.Saleur, Nucl. Phys. B376 (1992) 461

L.H.Kauffman and H.Saleur, Commun. Math. Phys. 141 (1991) 293

[5] A.Berkovich, C.G"mez and G.Sierra, "Spin-Anisotropy Commensurable Chains: Quantum Group Symmetries and N=2 SUSY", preprint IMAFF-93-1(1993)

[6] D.Bernard and A.LeClair, Phys. Lett. B247 (1990) 309

[7] P.Fendley and K.Intriligator, Nucl. Phys. B380 (1992) 265

[8] C.Fan and F.Y.Wu, Phys. Rev. B2 (1970) 723

[9] B.U.Felderhof, Physica 66 (1973) 279

[10] V.V.Bazhanov and Yu.G.Stroganov, Teor. Mat. Fiz. 62 (1985) 337

[11] E.H.Lieb, T.D.Schultz and D.C.Mattis, Ann. Phys.(NY) 16 (1961) 407

[12] H.Hinrichsen and V.Rittenberg, Phys. Lett. B275 (1992) 350

[13] E.K.Sklyanin, Func. Anal. Appl. 16 (1982) 263, Func. Anal. Appl. 17 (1983) 273

[14] I.V.Cherednik, Yad. Fiz. 36 (1982) 549, Fun. Anal. Appl. 19 (1985) 77

A.V.Odeski and B.L.Feigin, Func. Anal. Appl. 23 (1989) 207

[15] A.B.Zamolodchikov, Commun. Math. Phys. 69 (1979) 165

[16] A.B.Zamolodchikov, Sov. J. of Nucl. Phys. 46 (1987) 1090

C.Destri and H.J.de Vega, Mod. Phys. Lett. A4 (1989) 2595

[17] M.Toda, Phys. Rep. 18 (1975) 1 